\title{
Effective de Sitter brane via running radius
}

\author{
Nobuhiro Uekusa* \\ Department of Physics, \\ Kyushu University, Hakozaki, \\ Higashi-ku, Fukuoka 812-8581, Japan
}

\begin{abstract}
We present the solution for effective couplings on a running de Sitter brane. A renormalization group formalism is developed for linearized gravity in a two de Sitter brane model. It is shown that the effective tension remains a constant similarly to the zero cosmological constant case. We also discuss the suppression of higher derivative terms.
\end{abstract}

*uekusa@higgs.phys.kyushu-u.ac.jp 


\section{INTRODUCTION}

The holographic renormalization group plays an important role to gain a deep understanding of effective field theory. This is motivated by the AdS/CFT conjecture where the size of an extra dimension is identified with the renormalization group scale in dual field theory 1, 2, 3]. In particular, it becomes a useful tool when the calculation of field theory is complicated. Much attention has been attracted by the applications to phenomenologically interesting brane world context such as the Randall-Sundrum (RS) model [4, 5, 6, 7].

A renormalization group formalism has been developed for theories which have a fixed position of visible brane but a variable position of hidden brane [8, 9]. It simplifies the calculation of running couplings for localized operators generated by quantum corrections 10, 11]. These analyses were applied to the case where cosmological constants on branes are zero. Since the observation favors a positive cosmological constant [12] and 4d Newton potential on de Sitter (dS) branes has been confirmed [13], we are interested in the case where cosmological constants on branes are nonzero. The purpose of the present paper is to apply the renormalization group formalism to a model with two dS branes.

In this paper, we vary the distance between two dS branes located at fixed points of the orbifold $\mathrm{S}^{1} / \mathrm{Z}_{2}$, while we keep the same physics on the visible brane. The requirement that bulk classical field equations do not change leads to the variation of the coefficients of hidden brane operators. We give the exact solution for the coefficients of localized quadratic graviton using a derivative expansion, where the corresponding differential equation has the smooth zero cosmological constant limit. In particular, we find out the radius independent tension of the effective hidden brane. This is similar to the case of the RS branes [9]. We also confirm that higher derivative terms in the effective hidden brane action are suppressed compared to a localized Einstein-Hilbert term against a small variation of the radius.

The paper is organized as follows. In Section [II, we present the solution for couplings on effective dS brane. In Section III, we investigate the suppression of higher derivative terms. Summary and discussions are given in the final section.

\section{COUPLINGS ON EFFECTIVE DE SITTER BRANE}

We begin with the gravitational action,

$$
\begin{aligned}
S & =S_{\text {bulk }}+S_{\text {hid }}+S_{\text {vis }}, \\
S_{\text {bulk }} & =\frac{1}{2 \kappa^{2}} \int d^{4} x d y \sqrt{-g}(R-2 \Lambda), \\
S_{\text {hid }} & =-\tau_{h} \int d^{4} x \sqrt{-g_{h}}, \\
S_{\text {vis }} & =-\tau_{v} \int d^{4} x \sqrt{-g_{v}},
\end{aligned}
$$

where $\Lambda(<0), \kappa, \tau_{h}$ and $\tau_{v}$ denote the bulk cosmological constant, the five-dimensional gravitational coupling constant, the tensions of the hidden and visible branes, respectively. The hidden and visible branes are located at $y=y_{h}$ and $y=y_{v}$ which correspond to two fixed points of the orbifold $\mathrm{S}^{1} / \mathrm{Z}_{2}$. The determinants of the metrics in the bulk and on the hidden and visible branes are denoted as $g, g_{h}$ and $g_{v}$, respectively. 
For the action (2.1), the background solution with positive cosmological constants on the hidden and visible branes is given by ${ }^{1}$

$$
\begin{gathered}
d s^{2}=A^{2}(y)\left(-d t^{2}+a^{2}(t) \delta_{i j} d x^{i} d x^{j}\right)+d y^{2}, \\
A(y)=\frac{\sqrt{\lambda}}{\mu} \sinh \left[\mu\left(y_{H}-|y|\right)\right],
\end{gathered}
$$

where $\mu=\sqrt{-\Lambda / 6}$. The constant of integration $y_{H}$ is determined by the boundary condition $A\left(y_{h}\right)=1$. The feature of the background (2.5) is summerized as follows. The cosmological constants on the visible and hidden branes are written equally as

$$
\lambda=\left(\frac{\dot{a}}{a}\right)^{2}
$$

The terminology "dS brane" means a brane with positive cosmological constant $\lambda$. As a consistency condition, the cosmological constants on these branes are related to the parameters in the action (2.1),

$$
\lambda=\frac{\Lambda}{6}+\frac{\kappa^{4} \tau_{v}^{2}}{36} .
$$

In addition, the brane tensions are constrained as

$$
\tau_{h}=-\tau_{v}
$$

As seen from the warp factor (2.6), the location at $y=y_{H}$ is interpreted as a horizon. In the following, the region of the fifth dimension is taken as $y_{h} \leq y \leq y_{v}<y_{H}$.

We consider the gravitational fluctuation around the background (2.5),

$$
d s^{2}=A^{2}(y)\left(-d t^{2}+a^{2}(t)\left[\delta_{i j}+h_{i j}\left(x^{\mu}, y\right)\right] d x^{i} d x^{j}\right)+d y^{2},
$$

where $x^{\mu}=\left(t, x^{i}\right)$. This is convenient for the present calculations although in the flat case, the fluctuation is often written as $A^{2} \eta_{\mu \nu}+h_{\mu \nu}$ instead of $A^{2}\left(\eta_{\mu \nu}+h_{\mu \nu}\right)$. Under the gauge conditions, $h_{i}^{i}=0$ and $\partial_{i} h^{i j}=0$, the field equation derived from the equations (2.1) and (2.10) is given by

$$
\nabla_{M}^{2} h_{i j}=0
$$

where $\nabla_{M}$ denotes the five-dimensional covariant derivative. In the equation (2.11), we work with a system of only gravity and the distance between the two branes is not dynamical. We assume the proper radius is chosen so as to fit a possible explanation of the hierarchy as in the RS scenario [4]. Thus the fluctuation in the bulk is completely determined by the equation (2.11).

According to [8, 9], we shorten the distance between the two branes without varying the bulk field equation of the equation (2.11) with fixed $y_{v}$. Then effective local terms can be induced on the running hidden brane. We would like to derive the radius dependence of

${ }^{1}$ The localization of various bulk fields on a brane with a positive cosmological constant has been investigated for the background (2.5) 13, 14, 15]. 
these local terms. To investigate it, we introduce the effective hidden brane $S_{l}$ located at $y=l\left(\geq y_{h}\right)$. In other words, by replacing $S_{\text {hid }}$ in the action (2.1) with $S_{l}$, we obtain the effective theory in $l \leq y \leq y_{v}$,

$$
S_{\text {eff }}=S_{\text {bulk }}+S_{l}+S_{\text {vis }}
$$

As the fluctuation $h_{i j}$ is small, the quadratic action of the effective hidden brane at $y=l$ is given by

$$
S_{l}=-\tau_{h} \int d^{4} x \frac{1}{4} \alpha(x, l) \sqrt{-g_{l}}\left(A^{2} h\right)^{2},
$$

where $g_{l}$ denotes the determinant of the metric on the effective hidden brane. The $\alpha(x, l)$ stands for a series of couplings multiplied by derivative operators. In four-dimensional Fourier transforms $\alpha(x, l) \rightarrow \alpha(q, l)$, it is represented as

$$
\alpha(q, l)=\sum_{j=0,1, \cdots} \alpha^{(2 j)}(l)(q / A)^{2 j}
$$

where momentum $q$ is defined as a small dimensionless quantity divided by some large mass scale. In the equations (2.13) and (2.14), we omitted the explicit tensor structure and $a(t)$ dependence since we are particularly interested in the position dependence of the coupling $\alpha$.

The linearized field equation derived from the effective action $S_{\text {eff }}$ is given by ${ }^{2}$

$$
\left(A^{-2} \nabla_{\mu}^{2}+\partial_{y}^{2}+4 \frac{\left(\partial_{y} A\right)}{A} \partial_{y}-2 \kappa^{2} \tau_{h}(\alpha-1) \delta(y-l)\right) h=0 .
$$

From the equation (2.15), the boundary condition at $y=l$ is obtained as

$$
\left.\partial_{y} h(y)\right|_{y=l}=\kappa^{2} \tau_{h}(\alpha-1) h(l) .
$$

Combining this with the field equation (2.15) leads to the differential equation for $\alpha$,

$$
\frac{\partial}{\partial l} \alpha(l)=-\kappa^{2} \tau_{h}(\alpha-1)^{2}-4 \frac{A^{\prime}}{A}(\alpha-1)+\frac{q^{2}}{\kappa^{2} \tau_{h} A^{2}} .
$$

where $A=A(l)$ and ${ }^{\prime}=\partial / \partial l$. When we take the $\lambda \rightarrow 0$ limit $A=e^{-\mu l} \equiv 1 /(\mu z)$, the equation (2.17) reduces to

$$
z \frac{\partial}{\partial z} \alpha=-6(\alpha-1)^{2}+4(\alpha-1)+\frac{(q z)^{2}}{6}
$$

where we used $6 \mu=\kappa^{2} \tau_{h}$. This agrees with the equation in the flat case derived in [9].

We can find the exact solution for the equation (2.17),

$$
\alpha=1+\frac{2 \mu}{\kappa^{2} \tau_{h}} X \sqrt{1+X}\left[\frac{2}{X}-\frac{\frac{\beta_{+} \beta_{-}}{3} F\left(1+\beta_{+}, 1+\beta_{-}, 4 ;-X\right)-B G_{22}^{20}\left(\begin{array}{c|c}
-X & \begin{array}{c}
-\beta_{+} \\
-3
\end{array}
\end{array}\right)}{F\left(\beta_{+}, \beta_{-}, 3 ;-X\right)+B G_{22}^{20}\left(-X \mid \begin{array}{c}
1-\beta_{+} \\
-2
\end{array}\right)}\right]
$$

\footnotetext{
${ }^{2}$ In the flat case, the factor $(3 \alpha-4)$ of the equation $(3.7)$ in $[9]$ can be found in the equation for the rescaled fluctuation $\hat{h}=A^{2} h$, where $\partial_{y}^{2} h=4 \mu \delta(y-l) \hat{h} / A^{2}+$ (no delta functions) and $\kappa^{2} \tau_{h}=6 \mu$ are used.
} 


$$
X=\frac{1}{\sinh ^{2}\left[\mu\left(y_{H}-l\right)\right]}, \quad \beta_{ \pm}=\frac{1}{4}\left(5 \pm 3 \sqrt{1+\frac{4 q^{2}}{9 \lambda}}\right),
$$

where we introduced the new postion coordinate $X$ such that smaller $X$ is closer to the original hidden brane, while $X \rightarrow \infty$ corresponds to the horizon. The functions $F(\delta, \beta, \gamma ;-X)$ and $G_{22}^{20}\left(\begin{array}{l|ll}-X & \delta & \beta \\ \gamma & 0\end{array}\right)$ stand for the Gauss's hypergeometric function and Meijer's $G$-function, respectively. The constant of integration $B$ is determined by the boundary condition $\alpha\left(y_{h}\right)=1$ from the equation (2.11) and (2.15),

$$
B=-\frac{F\left(\beta_{+}, \beta_{-}, 3 ;-\frac{\lambda}{\mu^{2}}\right)-\frac{\beta_{+} \beta_{-}}{3} \frac{\lambda}{2 \mu^{2}} F\left(1+\beta_{+}, 1+\beta_{-}, 4 ;-\frac{\lambda}{\mu^{2}}\right)}{G_{22}^{20}\left(-\frac{\lambda}{\mu^{2}} \mid \begin{array}{cc|c}
1-\beta_{+} & 1-\beta_{-} \\
-2 & 0
\end{array}\right)+\frac{\lambda}{2 \mu^{2}} G_{22}^{20}\left(-\frac{\lambda}{\mu^{2}} \mid \begin{array}{cc}
-\beta_{+} & -\beta_{-} \\
-3 & 0
\end{array}\right)} .
$$

Fig 11 shows the behavior of our solution (2.19) for the coupling $\alpha$ against the position $X$ as well as the derivative operator $(q / A)^{2}$. The coupling $\left.\alpha\right|_{q^{2}=0}$ is independent of the position and the slope $\left.\partial_{\left(q^{2} / A^{2}\right)} \alpha\right|_{q^{2}=0}$ is nonzero except at $X(l)=X\left(y_{h}\right) \equiv X_{h}$. This means that the tension of the effective hidden brane, $\tau_{h} \alpha^{(0)}$, is constant and that the localized curvature term appears on the effective hidden brane. The radius independent behavior of the effective hidden brane tension is similar to the case of RS branes.

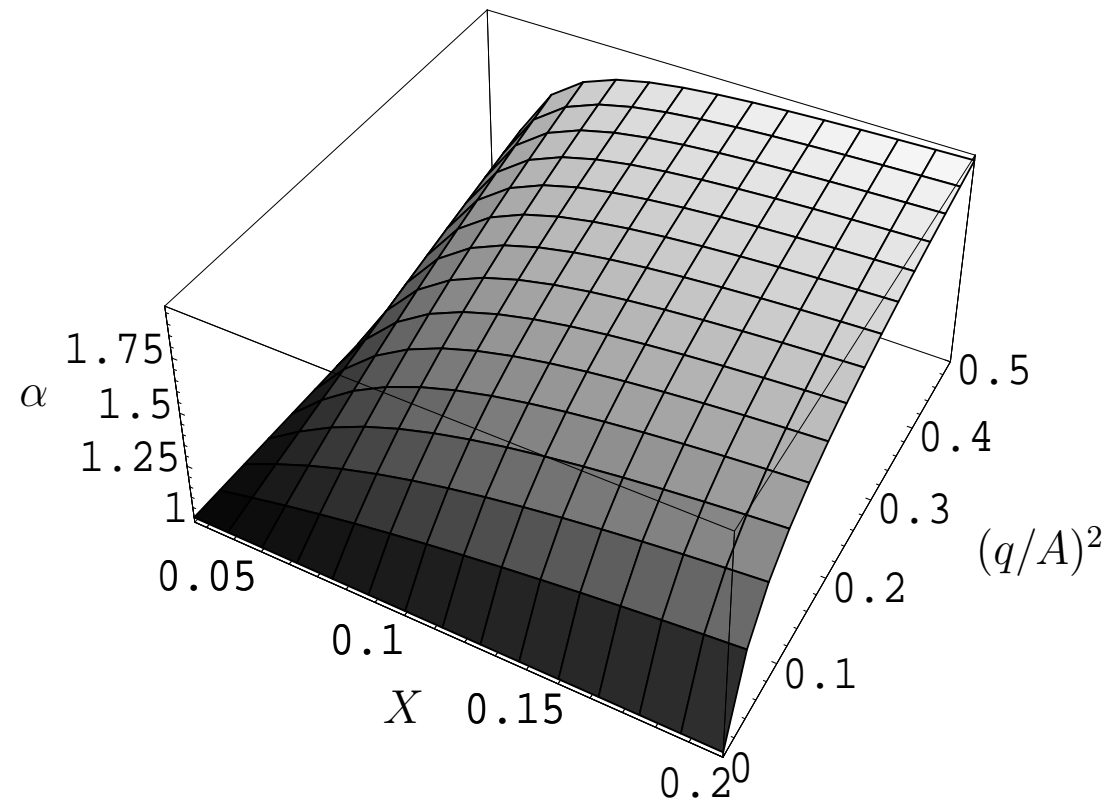

FIG. 1: $X$ - and $(q / A)^{2}$-dependences of the coupling $\alpha$, where $\kappa^{2} \tau_{h}=1.2, \mu=\sqrt{390} \times 10^{-2}$, $\lambda=10^{-3}$ and $X_{h}=1 / 39$.

It is useful to take some limits in order to study properties of the solution (2.19). We look at the coupling $\alpha$ in two limits, $q^{2}=0$ and large $X$.

For $q^{2}=0$, the equation (2.17) reduces to

$$
\frac{\partial}{\partial l} \alpha^{(0)}(l)=-\kappa^{2} \tau_{h}\left(\alpha^{(0)}-1\right)^{2}-4 \frac{A^{\prime}}{A}\left(\alpha^{(0)}-1\right) .
$$


The general solution for this equation is given by

$$
\alpha^{(0)}=1+\frac{3 \mu}{\kappa^{2} \tau_{h}} \frac{X^{2}}{(X-2) \sqrt{1+X}+C},
$$

where $C$ is a constant of integration. By imposing the boundary condition $\alpha^{(0)}\left(y_{h}\right)=1$, we obtain the solution,

$$
\alpha^{(0)}=1
$$

Thus the value of the hidden brane tension $\tau_{h}$ is preserved in the variation of the radius.

For a large $X$ limit, the coupling $\alpha$ is given by

$$
\alpha \simeq \frac{4 \mu}{\kappa^{2} \tau_{h}} \sqrt{X}\left(1-\mathcal{F}\left(q^{2}\right)\right)
$$

where $\mathcal{F}\left(q^{2}\right)$ denotes the coefficients dependent on $q^{2}$. Since $\mathcal{F}$ depends on $q^{2}$ only through

the form $\sqrt{1+4 q^{2} /(9 \lambda)}=\sqrt{1+4 q^{2} /\left(9 \mu^{2} X A^{2}\right)}$, we can identify a ratio of higher derivative terms,

$$
\alpha^{(2 j+2)}\left(\frac{q}{A}\right)^{2 j+2} / \alpha^{(2 j)}\left(\frac{q}{A}\right)^{2 j} \propto-\frac{q^{2}}{\mu^{2} X A^{2}}=-\frac{q^{2}}{\lambda}, \quad \text { for } j>0 .
$$

This result seems to imply that for tiny $\lambda$, the contributions of higher derivative terms in the action (2.13) can exceed the Einstein-Hilbert term (i.e. the $q^{2}$ term) far away from the original position of the hidden brane. However before concluding the breakdown of linearized approximation, one must require to take KK-modes into account since KK-modes are localized near the visible brane [16]. We leave this problem in future. In next section, we will study the contributions of higher derivative terms at $l \sim y_{h}$.

\section{THE SUPPRESSION OF HIGHER DERIVATIVE TERMS}

We would like to examine the radius dependence of $\alpha$ at each order of derivative expansion. We need to check particularly whether the contributions of higher derivative terms are negligible. Since it is difficult to calculate directly a derivative expansion of our solution (2.19), we return to the differential equation (2.17).

Expanding the equation (2.17) about $(q / A)^{2}$, we obtain a set of the equations as

$$
\begin{aligned}
& \alpha^{(2) \prime}+2 \frac{A^{\prime}}{A} \alpha^{(2)}+-\frac{1}{\kappa^{2} \tau_{h}}=0, \\
& \alpha^{(4) \prime}+\kappa^{2} \tau_{h} \alpha^{(2) 2}=0, \\
& \cdots,
\end{aligned}
$$

where the ellipsis corresponds to the equations for $(q / A)^{2 n}, n>2$. The equations in (3.1) are numerically solved. The position dependences of the couplings $\alpha^{(0)}, \alpha^{(2)}$ and $\alpha^{(4)}$ are shown in Fig, 2. For small $X$, higher derivative terms are of the order of lower derivative terms up to $\mathcal{O}\left((q / A)^{2}\right)$. Therefore as long as we consider a small variation of the radius, where $A \sim 1$ is satisfied, the contributions of higher derivative terms are suppressed by powers of $q^{2}$. 


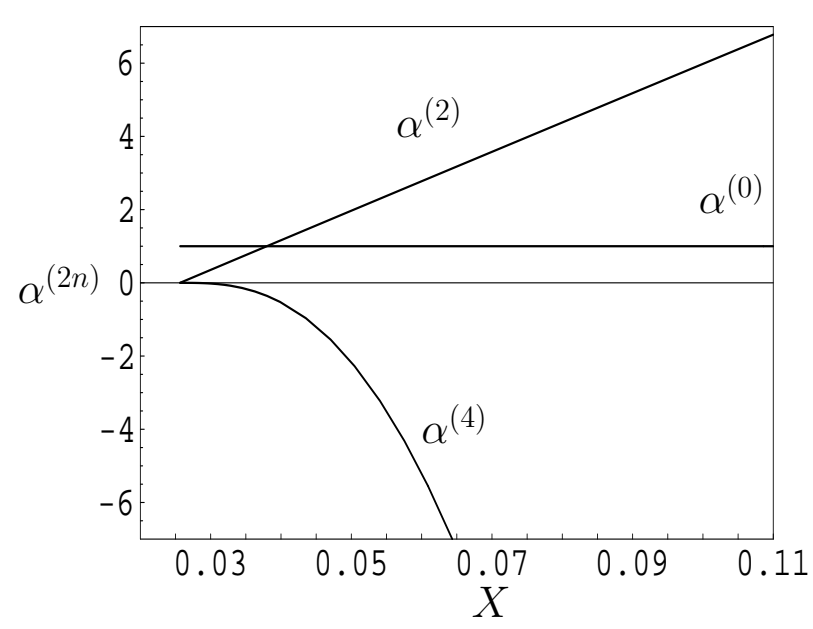

FIG. 2: $\alpha^{(2 n)}-X$ graph for $n \leq 2$, where $\kappa^{2} \tau_{h}=1.2, \mu=\sqrt{390} \times 10^{-2}, \lambda=10^{-3}$ and $X_{h}=1 / 39$.

\section{SUMMARY AND DISCUSSIONS}

In this paper, we have found the solution for effective couplings on the running dS brane for linearized gravity. The corresponding differential equation has the smooth $\lambda \rightarrow 0$ limit. The solution is a constant at zero-th order (corresponding to the effective tension) of a derivative expansion similarly to the case of a RS brane model. In addition, an EinsteinHilbert term and higher derivative terms appear in the effective hidden brane action with radius dependence. We have confirmed up to $\mathcal{O}\left(q^{6}\right)$ that higher derivative terms are suppressed against a small variation of the radius. Far away from the original position of the hidden brane, the KK-mode contribution becomes significant. As for KK-modes, it would be important to investigate not only the contributions of higher derivative terms but the spectrum of the KK gravitons which might be modified near the visible brane [17].

We finally note that the bulk background in our model is not AdS space in order to construct dS branes. Beyond the AdS/CFT conjecture, it remains to be examined whether some dual field theory exists. This would require explicit calculations of quantum corrections in field theory. If dual theory exists, it is worthwhile to examine whether and how conformal symmetry is broken.

\section{Acknowledgement}

The author thanks K. Yoshioka and K. Harada for reading the manuscript and discussions. He is also grateful to K. Ghoroku and K. Inoue for discussions.

[1] J. Maldacena, Adv. Theor. Math. Phys. 2, 231 (1998), Int. J. Theor. Phys. 38, 1113 (1999).

[2] S.S. Gubser, I.R. Klebanov and A.M. Polyakov, Phys. Lett. B 428, 105 (1998).

[3] E. Witten, Adv. Theor. Math. Phys. 2, 253 (1998). 
[4] L. Randall and R. Sundrum, Phys. Rev. Lett. 83, 3370 (1999); Phys. Rev. Lett. 83, 4690 (1999).

[5] H. Verlinde, Nucl. Phys. B 580, 264 (2000).

[6] V. Balasubramanian and P. Kraus, Phys. Rev. Lett. 83, 3605 (1999).

[7] S. Nojiri, S.D. Odintsov and S. Zerbini Phys. Rev. D 62, 064006 (2000);

M.J. Duff and J.T. Liu Phys. Rev. Lett. 85, 2052 (2000), Class. Quant. Grav. 18, 3207-3214, (2001);

S. Nojiri and S.D. Odintsov Phys. Lett. B 484, 119 (2000).

[8] A. Lewandowski, M.J. May and R. Sundrum, Phys. Rev. D 67, 024036 (2003).

[9] A. Lewandowski and M. Redi, hep-th/0305013.

[10] H. Georgi, A.K. Grant and G. Hailu, Phys. Lett. B 506, 207 (2001).

[11] G. Dvali, G. Gabadadze and M. Porrati, Phys. Lett. B 485, 208 (2000).

[12] C.L. Bennett, M. Halpern, G. Hinshaw, N. Jarosik, A. Kogut, M. Limon, S.S. Meyer, L. Page, D.N. Spergel, G.S. Tucker, E. Wollack, E.L. Wright, C. Barnes, M.R. Greason, R.S. Hill, E. Komatsu, M.R. Nolta, N. Odegard, H.V. Peiris, L. Verde and J.L. Weiland, astro-ph/0302207

[13] A. Kehagias and K. Tamvakis, Class. Quant. Grav. 19, L185 (2002);

S. Nojiri and S.D. Odintsov, Phys. Lett. B 548, 215 (2002);

K. Ghoroku, A. Nakamura and M. Yahiro, hep-th/0303068.

[14] I. Brevik, K. Ghoroku, S.D. Odintsov and M. Yahiro, Phys. Rev. D 66, 064016 (2002);

M. Ito hep-th/0204113,

P. Singh and N. Dadhich, hep-th/0208080.

[15] K. Ghoroku and N. Uekusa hep-th/0212102,

K. Ghoroku, M. Tachibana and N. Uekusa, hep-th/0304051.

[16] S.T. Giddings, E. Katz and L. Randall, J. High Energy Phys. 0003, 023 (2000).

[17] H. Davoudiasl, J.L. Hewett and T.G. Rizzo, hep-ph/0305086. 